\title{
Bacteremia due to anaerobic bacteria: epidemiology in a northern Bari Hospital, Italy
}

\author{
Maria Antonietta Distasi, Donatella Rizzi, Nicola Sciannandrone, Mirella Caldarone, \\ Simonetta Gioscia, Annamaria Leonetti, Tito Del Gaudio \\ Clinical Pathology Unit, Lorenzo Bonomo Hospital, ASL Andria, Italy
}

\section{Summary}

Background. Anaerobic bacteria are part of the commensal bacterial flora of skin and mucosae. Iatrogenic and pathological conditions altering this commensal relationship cause life-threatening diseases.

Materials and Methods. We analysed the blood cultures sent to the microbiology of our hospital between 2008 and the first quarter of 2013 to measure the frequency of bacteraemia caused by anaerobia. We examined 3138 vials of blood cultures for anaerobia, inoculated following in-house standard procedures. The colonies grown in absence of air were subjected to biochemical analysis. The MICs of metronidazole for 23 of the 26 organisms was tested.

Results. Twelve bacteria of the Bacteroides genus were identified, 9 Propionibacterium acnes, 1 Peptosctreptococcus micros, 1 Lactobacillus acidophilus, 1 Clostridium perfringens, 1 Prevotella oralis, 1 Eubacterium lentum.

Conclusions. The analysis of the results suggests that the incidence of cultures positive to anaerobia was constant across the years. We note that advanced age, altered mucocutaneous tropism, alterations to the oral and intestinal bacterial flora intensify the risk of anaerobial pathogenicity. The analysis of the metronidazole-determined MIC suggests that the intestinal anaerobic flora responds well

Correspondence: Tito Del Gaudio, UOC Patologia Clinica, Presidio Ospedaliero Lorenzo Bonomo, Andria ASL, Via Raffaello Sanzio 16, 76123 Andria (BT), Italy.

Tel.: $+39.0883-299212$ - Fax: $+39.0883-299118$.

E-mail: titodelgaudio@gmail.com

Key words: Bacteremia, anaerobic bacteria, metronidazole, Bacteroides, Propionibacterium.

Contributions: the authors contributed equally.

Conflict of interest: the authors declare no potential conflict of interest.

Received for publication: 30 September 2014.

Accepted for publication: 6 March 2015.

() Copyright M.A. Distasi et al., 2015

Licensee PAGEPress, Italy

Microbiologia Medica 2015; 30:4738

doi: $10.4081 / \mathrm{mm} .2015 .4738$

This article is distributed under the terms of the Creative Commons Attribution Noncommercial License (by-nc 3.0) which permits any noncommercial use, distribution, and reproduction in any medium, provided the original author(s) and source are credited. to therapy and prophylaxis with Metronidazole, while the anaerobic bacteria residing on skin and other mucosae are resistant. It is however hard to determine the clinical impact of anaerobic bacteremiae and their effect on the outcome of the patient, due to the scarcity of available clinical data.

\section{Introduction}

Anaerobic bacteria are known for surviving in environments without oxygen. They belong to the commensal bacterial flora of skin and mucosae. They are, specifically, main components of the so-called intestinal microbiota, which is responsible, together with the epithelium, intestinal epithelium, and immune system, of maintaining the integrity of the gastroenteric apparatus and preserving the health of the person as a whole (4). These bacteria live in equilibrium with the host, they serve metabolic and trophic functions, and contribute to regulating the immune action both systemically and on the mucosae level. Pathological and iatrogenic conditions which, modifying the tropism of tissues, alter the equilibrium of the anaerobic bacterial ecoflora and its commensal relationship with the epitelium-mucosae system, are responsible of local and systemic pathologies which may be lethal (2). There is no significant data, in literature, on bacteremia caused by anaerobic microorganisms: in previous studies, anaerobes are an uncommon cause for sepsis, prompting many authors to refrain from requesting blood cultures for anaerobes (6). Our microbiology laboratory, on the other hand, performs, as a default in the set of requested blood cultures, blood cultures for anaerobes at least on one vial. We have thus carried out an analysis of the blood cultures performed in the microbiology laboratory of our hospital between 2008 and the first semester of 2013 , to measure the rate of bacteremia caused by anaerobes and identify possible ethiopathogenetic risk factors.

\section{Materials and Methods}

In the period considered for this paper we examined 3138 vials of blood culture for anaerobes (Bact/Alert FN BioMerieux, Marcy l'Etoile, France) (224 in 2008, 263 in 2009, 416 in 2010, 699 in 2011, 967 in 2012,569 in the first semester of 2013) performed for 2151 patients (averaging 1.45 vials per patient) (Figure 1), 23/26 of those (88.46\%) was of age $>65 \mathrm{yr}$. (averaging $79.3 \mathrm{yr}$., range $66-93 \mathrm{yr}$.). The vials were inoculated on Enriched Chocolate Agar, Agar Mac Conkey, Mannitol salt Agar, Agar Sabouraud+chloramphenicol, MRSA selective Agar, all incubated at $35^{\circ} \mathrm{C}$ in $10 \% \mathrm{CO}_{2}$ atmosphere and on Enriched Chocolate Agar and Columbia CAN Agar incubated at $35^{\circ} \mathrm{C}$ in anaerobic atmosphere using the GasPak EZ system (Becton Dickinson, Franklin Lakes, 
New Jersey, USA). The colonies that developed only in anaerobic atmosphere were identified using the Rapid ID32A BioMerieux system and, starting 2013, with the VITEK2 BioMerieux system using the ANC card. For 23/26 microorganisms the MIC of Metronidazole was defined with the plate diffusion method, using E-test strips (BioMerieux) on Chocolate Muller-Hinton.

\section{Results}

Twenty-six patients were found positive for anaerobic bacteria with an average of $1.28 \%$ ( 2 in 2008, average $1.37 \% ; 3$ in 2009, average $1.42 \% ; 4$ in 2010 , average $1.42 \% ; 6$ in 2011, average $1.34 \% ; 6$ in 2012, average $0.90 \%$; 5 in the first semester of 2013, average 1.25\%) (Figure 2). 12 bacteria of the Bacteroides genus were identified, as well as 9 Propionibacterium acnes (P. acnes), 1 Peptosctreptococcus micros (P. micron), 1 Lactobacillus acidophilus (L. acidophilus), 1 Clostridium perfringens ( $C$. perfringens), 1 Prevotella oralis (P. oralis), 1 Eubacterium lentum (E. lentum) (Table 1). The MIC of Metronidazole measured for all strains of $P$. acnes, $P$. micros and $L$. acidophilus was $>256 \mathrm{mgc} / \mathrm{mL}$. The average of the MIC measured on Bacteroides was $0.28 \mathrm{mcg} / \mathrm{mL}$ (range $0.125-1 \mathrm{mcg} / \mathrm{L}$ ), while the MIC measured for $C$. perfringens, $P$. oralis, $E$. lentum were respectively $0.25 \mathrm{mcg} / \mathrm{L}, 0.064 \mathrm{mcg} / \mathrm{L}$, and $16 \mathrm{mcg} / \mathrm{L}$.

\section{Conclusions}

The analysis of our results shows that the percentage of blood cultures positive for anaerobes was constant in time, except for a small drop in 2012, despite the greater number of vials tested. We can confirm, in agreement with the current literature, that most cases of sepsis are caused by bacteria of the Bacteroides genus, whose habitat is prevalently the intestines. Following cases of sepsis caused by Bacteroides, we found a high incidence of events caused by P. acnes, a microorganism found mostly on skin, conjunctiva, oral cavity, and less frequently in the intestinal tract $(8,9,11)$. In the cases we observed, those patients who develop sepsis by anaerobes are mostly staying in the Medical Area, while we saw only a small percentage of cultures positive to anaerobes in the Surgical area. It is likely that this would be because in the surgical area, especially when performing abdominal surgery, patients are subject to a profilactic treatment with Metronidazole. On the other hand, in the medical area, incoming patients are long-stay, and their clinical condition is already compro- mised by chronic metabolic, vascular or neoplastic pathologies and by prolonged therapies. These patients are thus more susceptible to the pathogenic activation of commensal microorganisms. The age of the patients in our study is moreover cause of all those conditions common in geriatric age such as altered mucous and cutaneous tropism and modifications of the bacterial flora of intestines and the oral cavity. These condition likely predispose the patient for anaerobic pathogenicity. In addition to the previous observations, the analysis of the MIC of Metronidazole shows that, as reported by other authors, the anaerobic intestinal flora is always sensible to the therapy and to the prophylaxis with this drug, while the anaerobic flora which is prevalent on skin and other mucosae (P. acnes, $P$. micros, $L$. acidophilus) is resistant $(1,3)$. These observations prompt us to conclude, in agreement with other

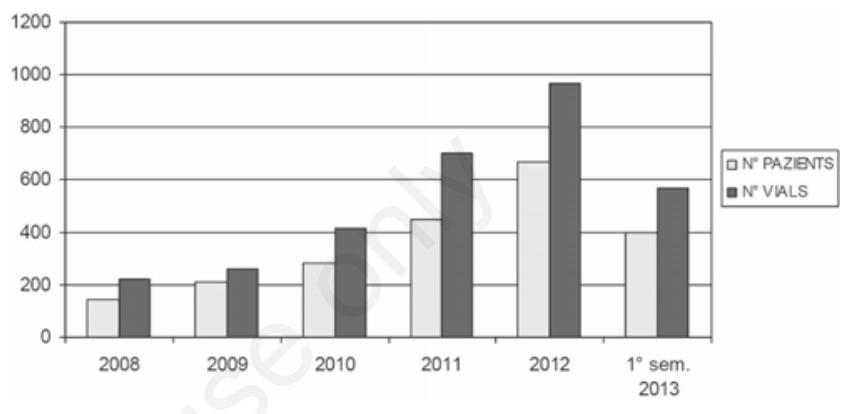

Figure 1. Number of blood culture for anaerobe bacteria $v s$ number of patients.

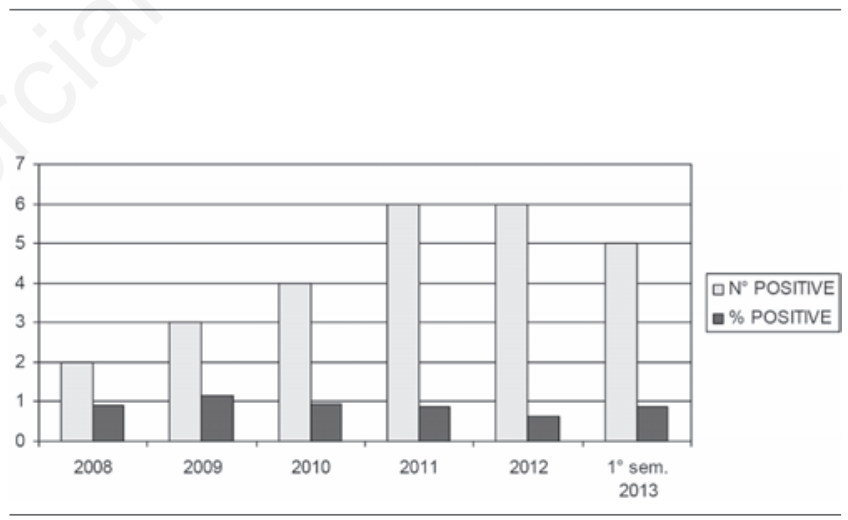

Figure 2. Number of blood cultures found positive for anaerobe bacteria.

Table 1. Anaerobe bacteria identified (total=26).

\begin{tabular}{|c|c|c|c|c|c|c|c|}
\hline & 2008 & 2009 & 2010 & 2011 & 2012 & Jan-March 2013 & Total \\
\hline P. acnes & 2 & 1 & 3 & 2 & 1 & - & 9 \\
\hline P. micros & - & - & - & - & 1 & - & 1 \\
\hline B. fragilis & - & 2 & 1 & 2 & - & 1 & 6 \\
\hline B. tetaiotaomicron & - & - & - & 1 & - & 2 & 3 \\
\hline B. ovatus & - & - & - & 1 & 1 & - & 2 \\
\hline B. distasonis & - & - & - & & 1 & - & 1 \\
\hline L. acidophilus & - & - & - & - & 1 & - & 1 \\
\hline C. perfringens & - & - & - & - & 1 & - & 1 \\
\hline Prevotella oralis & - & - & - & - & - & 1 & 1 \\
\hline Eubacterium lentum & - & - & - & - & - & 1 & 1 \\
\hline
\end{tabular}


authors, that bacteremia caused by anaerobic microorganisms develop in conditions that are connected with age, as well as pathological conditions such as blood dyscrasias and alterations of tissue microcirculation, which render the subject more susceptible to this kind of infection $(9,11)$. We cannot moreover exclude the possibility that, especially considering the high frequency of cultures positive to $P$. acnes, the anaerobes found be acting as a contaminating microorganism $(5,10)$. Unfortunately, the small size of our clinical data set does not let us establish with certainty the real clinical importance of bacteremia caused by anaerobes and of their contributions to the outcome of the patient $(2,6,7)$. We thus propose, in disagreement with some authors, to add vials for blood cultures for anaerobes, because we can only find what we constantly look for.

\section{References}

1. Brook I. Antimicrobial treatment of anaerobic infections. Expert Opin Pharmacother 2011; 12: 1691-707.

2. Brook I. The role of anaerobic bacteria in bacteremia. Anaerobe 2010; 16: 183-9.

3. Dubreuil L, Odou MF. Anaerobic bacteria and antibiotics: what kind of unexpected resistance could I find in my laboratory tomorrow? Anaerobe 2010; 16: 555-9.

4. Gasbarrini A. Il microbiota intestinale composizione, funzioni e patologie correlate. Il $4^{\circ}$ organo dell'apparato digerente. Verduci Editore 2013 IV-VI.

5. Hartzell JD, Torres D, Kim P, Wortmann G. Incidence of bacteremia after routine tooth brushing. Am J Med Sci 2005; 329: 178-80.

6. Iwata K, Takahashi M. Is anaerobic blood culture necessary? If so, who needs it? Am J Med Sci 2008; 336: 58-63.

7. Jirsa R, Marešová V, Brož Z. Current clinical significance of anaerobic bacteremia Klin Mikrobiol Infekc Lek 2010; 16: 158-63.

8. Kierzkowska M, Majewska A, Kqdzielska J, et al. Participation of gram-negative anaerobes in infections in hospitalized patients. Med Dosw Mikrobiol 2011; 63: 235-40.

9 Ngo JT, Parkins MD, Gregson DB, et al. Population-based assessment of the incidence, risk factors, and outcomes of anaerobic bloodstream infections. Infection 2013; 41: 41-8.

10 Park HJ, Na S, Park SY, et al. Clinical significance of Propionibacterium acnes recovered from blood cultures: analysis of 524 episodes. J Clin Microbiol 2011; 49: 1598-1601.

11. Wilson JR, Limaye AP. Risk factors for mortality in patients with anaerobic bacteremia. Eur J Clin Microbiol Infect Dis 2004; 23: $310-6$. 